\title{
Mouthguard-effect of high-intensity weight training on masticatory muscle tone and stiffness in taekwondo athletes
}

\author{
Joong-San Wang', Dae-Won Seo ${ }^{2}$, Jun-Youl Cha ${ }^{2, *}$ \\ 'Department of Physical Therapy, Howon University, Gunsan, Korea \\ 2Department of Sports \& Guard, Howon University, Gunsan, Korea
}

This study aimed to analyze the effect of wearing a mouthguard or not on masticatory muscle tone and stiffness of male taekwondo athletes during high-intensity weight training. The participants were male taekwondo athletes and aged 18 to 22 years. We randomly divided 24 men into the control group (CON; without a mouthguard; $n=12$ ) and the experimental group (MOG; with a mouthguard; $n=12$ ). Masticatory muscle tone and stiffness were measured after weight training at a level of $70 \%$ of one-repetition maximum. As a result of this study, masticatory muscle tone was no different in intragroup and intergroup after experiment. In addition, although the masticatory muscle stiffness was significantly higher in the dominant side of the MOG, there was no difference in intergroup, intragroup, and interaction. Through this study, it is necessary to consider the selective wearing of a mouthguard according to the training methods when partially analyzed from the physiological point of view of the masticatory muscle of taekwondo athletes.

Keywords: Mouthguard, Masticatory muscle tone, Masticatory muscle stiffness, Weight training

\section{INTRODUCTION}

The orofacial injuries have been increased in the combat sports or martial arts such as boxing, wushu, taekwondo etc. The prevention and management of orofacial injuries or dental trauma are very important parts of the medical care services for the sports athletes (dela Cruz et al., 2008; Garon et al., 1986; Tuna and Ozel, 2014). The mouthguard is used for the prevention and management of orofacial or dental injuries and temporomandibular joint disorders (Mizumachi et al., 2008). Recently, the combat sportsmen have used a mouthguard for conducting high-intensity training for improving the physical fitness including muscle strength and endurance (Mizumachi et al., 2008). In other words, although a mouthguard was originally used for orofacial injuries prevention, elite athletes are now using performance a mouthguard to improve athletic success. In the aspect of using a mouthguard, several researchers reported that the clenching a mouthguard has a positive effect on muscle strength and quickness, while some researchers reported that there was no difference of strength with or without using a mouthguard (Alghadir et al., 2017; Bates and Atkinson, 1983; Cetin et al., 2009; Garon et al., 1986; Yates et al., 1984).

Parker et al. (1984) found no significant differences in shoulder adduction and knee extension whether subjects were or were not wearing interocclusal splints. On the other hand, several researchers have studied the ergogenic effects of wearing a mouthguard on different functional and physiological strength and muscular power performances (Bergamini et al., 2008; Bernat et al., 2016). Moreover, some researchers suggested that the improvements in neuromuscular connections with jaw repositioning have been observed through improvements in muscle activation and proprioceptive function (Bernat et al., 2016). In other words, there is no consensus about the relationship between the use of a mouthguard and muscle strength. Especially, high-intensity training is required for athletes to perform weight training to improve athletic perfor-
${ }^{\star}$ Corresponding author: Jun-Youl Cha (D) https://orcid.org/0000-0003-0396-6217 Department of Sports \& Guard, Howon University, 64 Howondae 3-gil, Impi-myeon, Gunsan 54058, Korea

E-mail: roksfcha@hanmail.net

Received: September 25, 2020 / Accepted: October 14, 2020
This is an Open Access article distributed under the terms of the Creative Commons Attribution Non-Commercial License (https://creativecommons.org/licenses/by-nc/4.0/) which permits unrestricted non-commercial use, distribution, and reproduction in any medium, provided the original work is properly cited. 
mance and protect muscles (Cha and Jee, 2018). When performing high-intensity weight training, a mouthguard is worn to protect teeth as well as masticatory muscles. However, until now, studies have not conducted specific studies on whether it is beneficial to perform high-intensity training with a mouthguard or whether there is a problem even if high-intensity training is performed without wearing a mouthguard.

Therefore, the goal of this study was to investigate whether during high-intensity weight training revealed masticatory muscle tone and stiffness changes compared with and without wearing a mouthguard in taekwondo athlete. Through this study, we would like to represent the new physiological effect of masticatory muscles by wearing a mouthguard for weight training of taekwondo athletes.

\section{MATERIALS AND METHODS}

\section{Subjects}

The participants consisted of 24 healthy males who had no history of missing tooth, facial paralysis, malocclusion, orthodontic treatment, and cardiorespiratory disease. The subjects were taekwondo athletes who were participated in regular taekwondo training 6 days per week. We randomly divided into the control group (CON; without a mouthguard; $\mathrm{n}=12$ ) and the experimental group (MOG; with a mouthguard; $n=12$ ). The mouthguard used single upper maxillary mouthguard (Pine Tree SangMoo-SA, Seoul, Korea) of soft silicone materials. This study used a single upper maxillary guard without a breathing hole because forced expiratory volume in $1 \mathrm{sec}$ differed according to types of mouthguard and breathing hole (Francis and Brasher, 1991). This study was approved by Howon University Institutional Review Board for Human (041585-201906-HR-001-01). Before this study, subjects were informed of the experiment procedures and purpose of the study and we were required to provide informed consent. All subjects also agreed not to perform exercises that would aerobic or anaerobic exercise before the experiment were included. The general characteristics of the participants are shown in Table 1.

\section{Muscle tone and stiffness}

We measured the masticatory muscle tone and stiffness of the subjects using the Myometer (Myoton PRO, Myoton AS, Tallinn, Estonia). This Myometer is used to measure the muscle tone and stiffness by applying a mechanical impact to the muscle tissue with a force of 0.40 newton $(\mathrm{N})$. This Myometer can quickly measure muscle tone and stiffness with high levels of reliability (Aird et al.,
Table 1. Physical characteristic of the subjects

\begin{tabular}{lcccc}
\hline Variable & $\operatorname{CON}(\mathrm{n}=12)$ & $\mathrm{MOG}(\mathrm{n}=12)$ & $Z$ & $P$-value \\
\hline Age $(\mathrm{yr})$ & $19.67 \pm 1.07$ & $19.83 \pm 1.19$ & -0.366 & 0.755 \\
Height $(\mathrm{cm})$ & $71.29 \pm 9.17$ & $70.33 \pm 10.32$ & -0.405 & 0.713 \\
Weight $(\mathrm{kg})$ & $178.67 \pm 6.88$ & $176.58 \pm 5.63$ & -0.869 & 0.410 \\
$\begin{array}{l}\text { Dominant side }(\mathrm{n}) \\
\quad \text { Left:right }\end{array}$ & $1: 11$ & $2: 10$ & &
\end{tabular}

Values are presented as mean \pm standard deviation.

$\mathrm{CON}$, control group; MOG, mouthguard group.

2012). The muscles that were measured the temporal muscle and masseter muscle of both sides. To determine the dominant side and nondominant side of both facial masticatory muscles, subjects were asked to chew gum. The side that was more comfortable for the subjects to feel was the dominant side, and the side that was not the nondominant side was selected. Before the study, we performed skin markers on the highest point of the muscle belly of each measured muscle, and measured by placing the Myometer vertically on the skin marker. The muscle tone and stiffness of the bilateral anterior temporal muscle and masseter muscle were measured before and immediately after weight training.

\section{Experimental intervention}

We performed an exercise protocol, according to a previously study methods (Clark et al., 2019; dela Cruz et al., 2008). Prior to main workout, all subjects were performed warm-up and cool-down for $10 \mathrm{~min}$, respectively. The workout protocol consisted of seven types weight training (leg extension, squat on smith machine, leg curl, kick back, leg press, standing calf raises, and deadlift). The weight training for all subjects was repeated twelve times a set with $70 \%$ of one-repetition maximum (1RM), and a total of three sets were performed. The rest time between each set was $60 \mathrm{sec}$. The measurement of 1RM was based on the previous studies (Clark et al., 2019; Park et al., 2019).

\section{Statistical analysis}

Prior to analysis, we observed differences between groups using the Mann-Whitney $U$-test before comparing between groups, as shown in Table 1. All data are reported as the mean (standard deviation). The sample size was determined using $\mathrm{G}^{*}$ Power $\mathrm{v} 3.1 .3$, considering an a priori effect size of $\mathrm{f}^{2}(\mathrm{~V})=0.35$ (medium-size effect), $\alpha$ error probability of 0.05 , and power $(1-\beta$ error probability) of 0.95 . Based on the results of the Shapiro-Wilk test, the nonparametric Mann-Whitney $U$-test and Wilcoxon rank test were used to examine the differences of variables between groups 
and to examine the changes of variables between times. A significance of $P<0.05$ was employed. IBM SPSS Statistics ver. 22.0 (IBM Co., Armonk, NY, USA) was used for all analyses.

\section{RESULTS}

\section{Comparison with anthropometric indices between groups}

As shown in the Table 1, there were no differences in baseline characteristics between the CON and MOG, indicating that homogeneity was established.

\section{Difference and change of masticatory muscle tone and stiffness}

As shown in Table 2, the muscle tones of nondominant and dominant masticatory muscles in both groups tended to be increased after the experiment, although not significant different in intergroup. As shown in Table 3, the muscle stiffness of both masticatory muscles in the groups showed an overall increase as well,

Table 2. Comparative results of muscle tone $(\mathrm{Hz})$ of masticatory muscle

\begin{tabular}{lccccccc}
\hline & \multicolumn{3}{c}{ Nondominant } & & \multicolumn{3}{c}{ Dominant } \\
\cline { 2 - 3 } \cline { 6 - 8 } & CON & MOG & $Z$ & & CON & MOG & $Z$ \\
\hline Pre & $13.94 \pm 0.70$ & $14.05 \pm 1.11$ & -0.202 & & $13.59 \pm 0.58$ & $13.65 \pm 1.24$ & -0.087 \\
Post & $14.38 \pm 2.11$ & $14.41 \pm 1.13$ & -0.924 & & $14.38 \pm 1.98$ & $13.94 \pm 1.53$ & -0.404 \\
$Z$ & -0.311 & -1.646 & & & -1.512 & -0.863 & \\
\hline
\end{tabular}

Values are presented as mean \pm standard deviation.

CON, control group; MOG, mouthguard group. although not significant different in intergroup. Specifically, although the muscle stiffness of the nondominant masticatory muscle of the MOG was significantly increased compared with that $\mathrm{CON}(P<0.05)$, there was no significant difference in intergroup.

\section{Difference and change of temporal muscle tone and stiffness}

As shown in Table 4, the muscle tones of dominant and nondominant temporal muscles in both groups tended to be increased except for that of nondominant temporal muscle in the MOG after the experiment. As shown in Table 5, the muscle stiffness of both temporal muscles in the groups showed an overall increase as well except for that of nondominant temporal muscle in the MOG. Although the muscle tone and stiffness of two temporal muscles in the both groups were increased compared with those of $\mathrm{CON}$, there were no significant differences in intergroup and intragroup.

Table 4. Comparative results of muscle tone ( $\mathrm{Hz})$ of temporal muscle

\begin{tabular}{lccccccc}
\hline & \multicolumn{3}{c}{ Nondominant } & & \multicolumn{3}{c}{ Dominant } \\
\cline { 2 - 3 } \cline { 6 - 7 } & CON & MOG & $Z$ & & CON & MOG & $Z$ \\
\hline Pre & $29.04 \pm 4.53$ & $31.39 \pm 4.86$ & -1.415 & & $29.05 \pm 4.45$ & $31.32 \pm 5.05$ & -1.184 \\
Post & $29.35 \pm 4.95$ & $30.04 \pm 4.00$ & -0.808 & & $30.07 \pm 3.94$ & $32.61 \pm 4.93$ & -1.675 \\
$Z$ & -0.471 & -1.098 & & & -0.746 & -0.941 & \\
\hline
\end{tabular}

Values are presented as mean \pm standard deviation.

CON, control group; MOG, mouthguard group.

Table 3. Comparative results of muscle stiffness (Nm) of masticatory muscle

\begin{tabular}{|c|c|c|c|c|c|c|}
\hline \multirow{2}{*}{ Time } & \multicolumn{3}{|c|}{ Nondominant } & \multicolumn{3}{|c|}{ Dominant } \\
\hline & CON & MOG & $Z$ & CON & MOG & $Z$ \\
\hline Pre & $271.21 \pm 32.28$ & $270.79 \pm 51.26$ & -0.318 & $262.75 \pm 32.97$ & $265.79 \pm 33.70$ & -0.520 \\
\hline Post & $278.46 \pm 75.50$ & $295.92 \pm 62.04$ & -1.155 & $303.00 \pm 109.94$ & $273.71 \pm 55.61$ & -0.289 \\
\hline$Z$ & -0.432 & $-1.961^{*}$ & & -1.778 & -0.314 & \\
\hline
\end{tabular}

Values are presented as mean \pm standard deviation.

$\mathrm{CON}$, control group; $\mathrm{MOG}$, mouthguard group.

${ }^{*} P<0.05$.

Table 5. Comparative results of muscle stiffness (Nm) of temporal muscle

\begin{tabular}{|c|c|c|c|c|c|c|}
\hline \multirow{2}{*}{ Time } & \multicolumn{3}{|c|}{ Nondominant } & \multicolumn{3}{|c|}{ Dominant } \\
\hline & CON & MOG & $Z$ & CON & MOG & $Z$ \\
\hline Pre & $699.08 \pm 72.10$ & $731.00 \pm 109.30$ & -1.039 & $683.46 \pm 74.50$ & $743.92 \pm 96.04$ & -1.501 \\
\hline Post & $712.21 \pm 84.73$ & $711.63 \pm 64.86$ & -0.346 & $715.04 \pm 58.92$ & $754.25 \pm 87.25$ & -1.126 \\
\hline$Z$ & -0.235 & -0.784 & & -1.648 & -0.549 & \\
\hline
\end{tabular}

Values are presented as mean \pm standard deviation.

CON, control group; MOG, mouthguard group. 


\section{DISCUSSION}

This present study found that the muscle tone and stiffness of masticatory muscle in the two groups showed the increased tendencies after the high-intensity weight training. However, there was no statistically significant difference between MOG and $\mathrm{CON}$ after the experiment. These tendencies represented in the dominant and nondominant sides of temporal muscles in both groups as well.

Mouthguard is a widely used combat sport athletes, but previous studies have mostly conclusions about the safety of teeth (dela Cruz et al., 2008) or training effect (Ball et al., 2011; Kim et al., 2018). Especially, in the aspect of training effectiveness, wearing a mouthguard is very important to the combat sportsmen. In fact, when neural firing of alpha motor neuron, muscle contraction begins (Stifani, 2014) and muscle tone can be increased (Ahmed, 2016). However, an excessive increase of muscle tone or stiffness can increase the risk of injury (Butler et al., 2003). From this point of view considering these negative effects, we began a study to find out how weight training after wearing a mouthguard affects the tone and stiffness of the masticatory muscles. Through this study, it is newly suggested that wearing a mouthguard may reduce the burden on the rigidity of the masticatory muscle when weight training is performed at $70 \%$ of $1 \mathrm{RM}$ in taekwondo athletes. Raquel et al. (2017) analyzed masticatory muscle activity using electromyography for the effect of wearing a mouthguard during training of Karate athletes. In their study, they observed the muscle activity of the both masticatory muscles in resting conditions after training. In their results, they reported that there was no significant difference between groups.

Masticatory muscles are clenched and chewing with neuromuscular control of the trigeminal nerve, but masticatory muscle fibers are type II muscle fibers predominate (temporal muscle: $52 \%$ to $73 \%$, masseter muscle: $48 \%$ to $65 \%$ ), this muscle activity decreases due to rapid muscle fatigue (Mao et al., 1992). Conversely, in healthy adults, continuous muscular contraction of specific muscles immediately increased muscle tone and stiffness significantly (Wang, 2017). Therefore, the changes in muscle activity and stiffness of connective tissue after training may have opposite results. In addition, the previous study has the disadvantage of analyzing the measured muscles with the left and right muscles without classifying them into dominant and nondominant muscles during chewing activity. Our research has a feature that complements the disadvantages of previous research by dividing it into the dominant side and the nondominant side during mastication activity, so it can be more advantageous in the use of research results.

Considering the characteristics of combat sport athletes, it may be necessary to wear a mouthguard during weight training due to problems with the jaw joint or teeth. We also recommended wearing a mouthguard in taekwondo athletes and performing a massage or a stretching on the masticatory muscles to reduce increased stiffness in the nondominant or dominant side after weight training. Actually, the rehabilitation program combined with massage and transcutaneous electrical nerve stimulation is effective in reducing muscle tone and stiffness caused by continuous strong muscle contraction (Nakamura et al., 2018; Wang, 2017). Moreover, the high-intensity weight training for taekwondo athletes is very important for their successful competition. In other words, after weight training of combat sport athletes, the masticatory muscle tone and stiffness have been managed for their future. These results of this study indicate that the teeth alignment and occlusal force are improved after weight training is performed while wearing the mouthguard and the muscle contraction force is increased in the nondominant muscle. In addition, it is thought that the muscle contraction, which unconsciously bites the mouthguard while performing weight training, continuously repeated muscle contraction increased muscle tone of the nondominant masticatory muscle, although not significant. In other words, although the masticatory muscle tone and stiffness were higher in the dominant or nondominant sides of MOG compared with those of $\mathrm{CON}$, there was no difference in intergroup, intragroup, and interaction. Through this study, it is not necessary to consider wearing of a mouthguard when taekwondo athletes should perform a weight training at $70 \%$ of $1 \mathrm{RM}$.

Considering the characteristics of combat sport athletes, it may be necessary to wear a mouthguard during weight training due to problems with the jaw joint or teeth (Castroflorio et al., 2005). Dunn-Lewis et al. (2012) reported that wearing a mouthguard during the power balance performance improves performance of upper-body loaded power exercises in both men and women. On the other hand, Cetin et al. (2009) reported that there was no difference in 20-m sprint time, jumping tests, handgrip strength, isometric leg, and back strength in comparison of exercise performance according to the presence or absence of wearing a mouthguard of taekwondo players. However, they suggested that wearing a mouthguard could increase anaerobic performance and increase the isokinetic peak torque of the hamstring muscles. On dated, only some studies published to masticatory muscle activity while with or without wearing a mouthguard were found in the literature (Ginszt et al., 2020; Raquel et al., 2017). Wearing mouth- 
guard can be expected to modulate unnecessary masticatory muscle activity of combat sports athlete (Raquel et al., 2017). Although we have recommended wearing a mouthguard in taekwondo athletes and performing high-intensity weight training, this study confirms that it is not necessary to wear a mouthguard for weight training.

Eventually, as a result of this study, masticatory muscle tone was no different in intragroup and intergroup after experiment. In addition, although the masticatory muscle stiffness was significantly higher in the dominant side of the MOG, there was no difference in intergroup, intragroup, and interaction. Through this study, it is not necessary to consider wearing of a mouthguard when taekwondo athletes should perform a weight training at $70 \%$ of 1RM.

\section{CONFLICT OF INTEREST}

No potential conflict of interest relevant to this article was reported.

\section{ACKNOWLEDGMENTS}

This study was supported by the Research Grant of Howon University in 2020.

\section{REFERENCES}

Ahmed Z. Modulation of gamma and alpha spinal motor neurons activity by trans-spinal direct current stimulation: effects on reflexive actions and locomotor activity. Physiol Rep 2016;4:e12696.

Aird L, Samuel D, Stokes M. Quadriceps muscle tone, elasticity and stiffness in older males: reliability and symmetry using the MyotonPRO. Arch Gerontol Geriatr 2012;55:e31-e39.

Alghadir A, Zafar H, Iqbal ZA. Effect of upright and slouch sitting postures and voluntary teeth clenching on hand grip strength in young male adults. J Back Musculoskelet Rehabil 2017;30:961-965.

Ball N, Nolan E, Wheeler K. Anthropometrical, physiological, and tracked power profiles of elite taekwondo athletes 9 weeks before the Olympic competition phase. J Strength Cond Res 2011;25:2752-2763.

Bates Jr RE, Atkinson WB. The effects of maxillary MORA's on strength and muscle efficiency tests. J Craniomandib Pract 1983;1:37-42.

Bergamini M, Pierleoni F, Gizdulich A, Bergamini C. Dental occlusion and body posture: a surface EMG study. Cranio 2008;26:25-32.

Bernat B, Jose M, Mònica ST, Adrià M, Mario G. Effects of jaw clenching while wearing a customized bite-aligning mouthpiece on strength in healthy young men. J Strength Cond Res 2016;30:1102-1110.

Butler RJ, Crowell HP 3rd, Davis IM. Lower extremity stiffness: implications for performance and injury. Clin Biomech (Bristol, Avon) 2003; 18:511-517.

Castroflorio T, Farina D, Bottin A, Piancino MG, Bracco P, Merletti R. Surface EMG of jaw elevator muscles: effect of electrode location and inter-electrode distance. J Oral Rehabil 2005;32:411-417.

Cetin C, Keçeci AD, Erdoğan A, Baydar ML. Influence of custom-made mouth guards on strength, speed and anaerobic performance of taekwondo athletes. Dent Traumatol 2009;25:272-276.

Cha JY, Jee YS. Wushu Nanquan training is effective in preventing obesity and improving heart function in youth. J Exerc Rehabil 2018;14:466472.

Clark NC, Reilly LJ, Davies SC. Intra-rater reliability, measurement precision, and inter-test correlations of 1RM single-leg leg-press, knee-flexion, and knee-extension in uninjured adult agility-sport athletes: considerations for right and left unilateral measurements in knee injury control. Phys Ther Sport 2019;40:128-136.

dela Cruz GG, Knapik JJ, Birk MG. Evaluation of mouthguards for the prevention of orofacial injuries during United States Army basic military training. Dent Traumatol 2008;24:86-90.

Dunn-Lewis C, Luk HY, Comstock BA, Szivak TK, Hooper DR, Kupchak BR, Watts AM, Putney BJ, Hydren JR, Volek JS, Denegar CR, Kraemer WJ. The effects of a customized over-the-counter mouth guard on neuromuscular force and power production in trained men and women. J Strength Cond Res 2012;26:1085-1093.

Francis KT, Brasher J. Physiological effects of wearing mouthguards. Br J Sports Med 1991;25:227-31.

Garon MW, Merkle A, Wright JT. Mouth protectors and oral trauma: a study of adolescent football players. J Am Dent Assoc 1986;112:663665.

Ginszt M, Zieliński G, Byś A, Gawda P, Majcher P. Masticatory muscles activity in sport climbers. Int J Environ Res Public Health 2020;17:1378.

Kim KJ, Song HS, Yoon DH, Fukuda DH, Kim SH, Park DH. The effects of 10 weeks of $\beta$-alanine supplementation on peak power, power drop, and lactate response in Korean national team boxers. J Exerc Rehabil 2018;14:985-992.

Mao J, Stein RB, Osborn JW. The size and distribution of fiber types in jaw muscles: a review. J Craniomandib Disord 1992;6:192-201.

Mizumachi M, Sumita Y, Ueno T. Effect of wearing a mouthguard on the vestibulocollic reflex. J Sci Med Sport 2008;11:191-197.

Nakamura M, Hirabayashi R, Ohya S, Aoki T, Suzuki D, Shimamoto M, Kikumoto T, Ito W, Nakamura E, Takabayashi T, Edama M. Effect of static stretching with superficial cooling on muscle stiffness. Sports Med Int Open 2018;2:E142-E147. 
Park KM, Park SC, Kang S. Effects of resistance exercise on adipokine factors and body composition in pre- and postmenopausal women. J Exerc Rehabil 2019;15:676-682.

Parker MW, Pelleu Jr GB, Blank LW, Breton RW. Muscle strength related to use of interocclusal splints. Gen Dent 1984;32:105-109.

Raquel G, Namba EL, Bonotto D, Ribeiro Rosa EA, Trevilatto PC, Naval Machado MÂ, Vianna-Lara MS, Azevedo-Alanis LR. The use of a custom-made mouthguard stabilizes the electromyographic activity of the masticatory muscles among Karate-Dō athletes. J Bodyw Mov Ther 2017;21:109-116.
Stifani N. Motor neurons and the generation of spinal motor neuron diversity. Front Cell Neurosci 2014;8:293.

Tuna EB, Ozel E. Factors affecting sports-related orofacial injuries and the importance of mouthguards. Sports Med 2014;44:777-783.

Wang JS. Therapeutic effects of massage and electrotherapy on muscle tone, stiffness and muscle contraction following gastrocnemius muscle fatigue. J Phys Ther Sci 2017;29:144-147.

Yates JW, Koen TJ, Semenick DM, Kuftinec MM. Effect of a mandibular orthopedic repositioning appliance on muscular strength. J Am Dent Assoc 1984;108:331-333. 